\title{
Analysis of the Fractional-Order Kaup-Kupershmidt Equation via Novel Transforms
}

\author{
Naveed Iqbal $\mathbb{D}^{1},{ }^{1}$ Humaira Yasmin $\mathbb{D}^{2},{ }^{2}$ Ali Rezaiguia, ${ }^{1,3}$ Jeevan Kafle $\mathbb{D}^{\mathbb{D}},{ }^{4}$ \\ A. Othman Almatroud, ${ }^{1}$ and Taher S. Hassan ${ }^{1,5}$ \\ ${ }^{1}$ Department of Mathematics, Faculty of Science, University of Ha'il, Ha'il 2440, Saudi Arabia \\ ${ }^{2}$ Department of Basic Sciences, Preparatory Year Deanship, King Faisal University, Al-Ahsa 31982, Saudi Arabia \\ ${ }^{3}$ Department of Computer Science and Mathematics, Mouhamed Cherif Messadia University, Souk Ahras, Algeria \\ ${ }^{4}$ Central Department of Mathematics, Tribhuvan University Kritipur, Kathmandu, Nepal \\ ${ }^{5}$ Department of Mathematics, Faculty of Science, Mansoura University, Mansoura 35516, Egypt
}

Correspondence should be addressed to Naveed Iqbal; n.iqbal@uoh.edu.sa and Jeevan Kafle; jeevan.kafle@cdmath.tu.edu.np

Received 27 September 2021; Revised 2 November 2021; Accepted 8 December 2021; Published 26 December 2021

Academic Editor: Fairouz Tchier

Copyright $\odot 2021$ Naveed Iqbal et al. This is an open access article distributed under the Creative Commons Attribution License, which permits unrestricted use, distribution, and reproduction in any medium, provided the original work is properly cited.

\begin{abstract}
In this article, we develop a technique to determine the analytical result of some Kaup-Kupershmidt equations with the aid of a modified technique called the new iteration transform method. This technique is a mixture of the novel integral transformation Elzaki transformation and the new iteration technique. The nonlinear term can be handled easily by a new iteration technique. The results show that the combination of the Elzaki transformation and the new iteration technique is quite capable and basically well suited for applying in such problems and that it can be implemented to other nonlinear models. This technique is viewed as an effective alternative approach to certain existing approaches for such accurate models.
\end{abstract}

\section{Introduction}

Fractional calculus is regarded as an important branch of science, particularly for phenomena that cannot be defined by basic nonlinear ordinary differential equations or partial differential equations with integer-order operators. The use of memory is one of the main advantages of fractional-order derivatives over standard derivatives. In recent years, there have been numerous applications of fractional-order ordinary and partial differential equations in many fields of physics and engineering. There have been several key works discovered, particularly in genetic mechanics and in the viscoelasticity concept, where fractional-order derivatives are utilized for a good explanation of the properties of materials. This is the main benefit of fractional derivatives compared with traditional integer-order models in which such effects are neglected. The computational modeling and analysis of structures and procedures, based on the explanation of their properties in concepts of fractional derivatives, obviously result in differential equations of fractional order and the requirement of finding solutions such as mathematical equations [1-10].

The fractional-order Kaup-Kupershmidt equation is used to investigate the analysis of capillary gravity waves' attitude and nonlinear dispersive waves. The extensive fifthorder nonlinear development equation is written as

$$
D_{\tau}^{\rho} \mu(\zeta, \tau)+\alpha \mu \mu_{\zeta \zeta \zeta}+\beta p \mu_{\zeta} \mu_{\zeta \zeta}+\gamma \mu^{2} \mu_{\zeta}+\mu_{\zeta \zeta \zeta \zeta \zeta}=0,
$$

with the initial condition

$$
\mu(\zeta, 0)=g(\zeta),
$$

where $\alpha, \beta$, and $\gamma$ are real constants and $0<\rho \leq 1$ is the parameter symbolizing the order of the fractional-order derivative. By considering different values for $\alpha, \beta$, and $\gamma$, the overload nonlinear fifth-order development model can be scaled down to the fifth-order fractional-order Kaup-Kupershmidt equation.

For $\alpha=-15, \beta=-15$, and $\gamma=45$, the above equation simplifies to 


$$
D_{\tau}^{\rho} \mu(\zeta, \tau)-15 \mu \mu_{\zeta \zeta \zeta}-15 p \mu_{\zeta} \mu_{\zeta \zeta}+45 \mu^{2} \mu_{\zeta}+\mu_{\zeta \zeta \zeta \zeta \zeta}=0
$$

with the initial condition

$$
\mu(\zeta, 0)=g(\zeta)
$$

In 1980, Kaup [11] first introduced a significant dispersive basic Kaup-Kupershmidt equation, and then it was improved by Kupershmidt [12] in 1994. This study is concerned with the analysis of the modified fractionalorder Kaup-Kupershmidt (KK) equation. In recent decades, excellent scientific work has been devoted to the analysis of the classical $\mathrm{KK}$ equation. The modern $\mathrm{KK}$ equation can be integrated at $p=5 / 2[13]$ and is considered to have bilinear representation [14]. Soliton and solitary wave results can be obtained for general nonlinear development problems by importing four diverse techniques autonomously. Nonlaopon et al. [15] used the inverse scattering approach to establish soliton results to analyze nonlinear equations with physical implications. Two integrable differential-difference equations exhibit soliton solutions of the Kaup-Kupershmidt equation type [16]. Musette introduced the fifth-order KK equation, and Verhoeven was one of the combined instances of the Henon-Heiles method; see [17] for more details. Prakasha et al. [18] used the $q$-homotopy analysis transform method which is implemented to obtain the result for the fractionalorder KK equation.

Daftardar-Gejii and Jafari [19] introduced a new iterative methodology for investigating nonlinear equations in 2006. Jafari [20] was the first to use the Laplace transform in an iterative technique. In [21], Jafari et al. suggested a modified straightforward methodology, named iterative Laplace transformation technique, to look for the numerical effects of the fractional partial differential equation system. Iterative Laplace transformation technique is used to solve linear and nonlinear partial differential equations such as time-fractional Zakharov-Kuznetsov equation [22], fractional-order Fokker-Planck equation [23], and Fornberg-Whitham equation [24].

This article modified the iterative method with the Elzaki transform; the novel approach is named the iterative transformation technique. The new iterative transformation technique is implemented to evaluate the fractional order of the system of the KK equation. The outcome of several illustrative cases is described to demonstrate the effectiveness of the proposed technique. The present method is used to obtain the results of fractional-order and integral-order models. The new method reduces computing costs while increasing rate convergence. The proposed method is also helpful in dealing with other fractional-order linear and nonlinear partial differential equations.

\section{Basic Definitions}

Definition 1 (see [25-27]). The fractional-order Riemann-Liouville operator $D^{\rho}$ of order $\rho$ is defined as

$$
D^{\rho} \nu(\zeta)= \begin{cases}\frac{d^{\kappa}}{d \zeta^{\kappa}} \nu(\zeta), & \text { for } \rho=\kappa \\ \frac{1}{\Gamma(\kappa-\rho)} \frac{d}{d \zeta^{\kappa}} \int_{0}^{\zeta} \frac{\nu(\zeta)}{(\zeta-\psi)^{\rho-\kappa+1}} \mathrm{~d} \psi, & \text { for } \kappa-1<\rho<\kappa\end{cases}
$$

where $\kappa \in \mathbb{Z}^{+}, \rho \in \mathbb{R}^{+}$, and

$$
D^{-\rho} \nu(\zeta)=\frac{1}{\Gamma(\rho)} \int_{0}^{\zeta}(\zeta-\psi)^{\rho-1} \nu(\psi) \mathrm{d} \psi, \quad 0<\rho \leq 1
$$

Definition 2 (see [25-27]). The Riemann-Liouville fractional integral operator $J^{\rho}$ is given as

$$
J^{\rho} \nu(\zeta)=\frac{1}{\Gamma(\rho)} \int_{0}^{\zeta}(\zeta-\psi)^{\rho-1} \nu(\zeta) \mathrm{d} \zeta, \quad \zeta>0, \rho>0
$$

Some properties of the operator are as follows:

$$
\begin{aligned}
J^{\rho} \zeta^{\kappa} & =\frac{\Gamma(\kappa+1)}{\Gamma(\kappa+\rho+1)} \zeta^{\kappa+\psi}, \\
D^{\rho} \zeta^{\kappa} & =\frac{\Gamma(\kappa+1)}{\Gamma(\kappa-\rho+1)} \zeta^{\kappa-\psi} .
\end{aligned}
$$

Definition 3 (see [25-27]). The fractional-order Caputo operator ${ }^{C} D^{\rho}$ of $\rho$ is given as

$$
{ }^{C} D^{\rho} \nu(\zeta)= \begin{cases}\frac{1}{\Gamma(\kappa-\rho)} \int_{0}^{\zeta} \frac{\nu^{\kappa}(\psi)}{(\zeta-\psi)^{\rho-\kappa+1}} \mathrm{~d} \psi, & \text { for } \kappa-1<\rho<\kappa, \\ \frac{d^{\kappa}}{d \zeta^{\kappa}} \nu(\zeta), & \text { for } \kappa=\rho .\end{cases}
$$

Definition 4 (see [25-27]).

$$
\begin{aligned}
& J_{\zeta}^{\rho} D_{\zeta}^{\rho} g(\zeta)=g(\zeta)-\sum_{k=0}^{m} g^{k}\left(0^{+}\right) \frac{\zeta^{k}}{k !}, \quad \text { for } \zeta>0 \text { and } \kappa-1<\rho \leq \kappa, \kappa \in \mathbb{N}, \\
& D_{\zeta}^{\rho} J_{\zeta}^{\rho} g(\zeta)=g(\zeta) .
\end{aligned}
$$

Definition 5 (see [25-27]). The Elzaki transformation of the fractional Caputo derivative is expressed as

$$
E\left[D_{\zeta}^{\rho} g(\zeta)\right]=s^{-\rho} E[g(\zeta)]-\sum_{k=0}^{\kappa-1} s^{2-\rho+k} g^{(k)}(0)
$$

where $\kappa-1<\rho<\kappa$.

Definition 6 (see [25-27]). The inverse Elzaki transform is given as 
$E^{-1}[\mathfrak{T}(s)]=h(\mathfrak{I})=\frac{1}{2 \pi \iota} \int_{\alpha-\iota \infty}^{\alpha+\iota \infty} h\left(\frac{1}{s}\right) e^{s \Im} s \mathrm{~d} s=\sum$ residues of $h\left(\frac{1}{s}\right) e^{s \mathfrak{I}} s$

The inverse Elzaki transform of some of the functions is given by

$$
\begin{aligned}
\bullet E^{-1}\left\{s^{n}\right\} & =\frac{\mathfrak{J}^{n-2}}{(n-2) !}, n=2,3,4, \ldots, \\
\bullet E^{-1}\left(\frac{s^{2}}{1-a s}\right) & =e^{a \mathfrak{I}} \\
\bullet E^{-1}\left(\frac{s^{3}}{1+a^{2} s^{2}}\right) & =\frac{1}{a} \sin a \mathfrak{J} \\
\bullet E^{-1}\left(\frac{s^{2}}{1+a^{2} s^{2}}\right) & =\frac{1}{a} \cos a \mathfrak{J} .
\end{aligned}
$$

\section{The General Discussion of the Proposed Method}

Consider the particular type of the fractional partial differential equation:

$$
D_{\tau}^{\rho} v(\zeta, \tau)+M v(\zeta, \tau)+N v(\zeta, \tau)=h(\zeta, \tau), \quad n-1<\rho \leq n,
$$

where $n \in \mathbb{N}, M$ and $N$ are linear and nonlinear functions, and $h$ is a source function.

The initial condition is

$$
v^{k}(\zeta, 0)=g_{k}(\zeta), \quad k=0,1,2, \ldots, n-1 .
$$

Applying the Elzaki transform of (14), we obtain as $E\left[D_{\tau}^{\rho} v(\zeta, \tau)\right]+E[M v(\zeta, \tau)+N v(\zeta, \tau)]=E[h(\zeta, \tau)]$

The differentiation property is defined as
$E[v(\zeta, \tau)]=\sum_{k=0}^{m} s^{2-\rho+k} u^{(k)}(\zeta, 0)+s^{\rho} E[h(\zeta, \tau)]-s^{\rho} E[M v(\zeta, \tau)+N v(\zeta, \tau)]$

using the inverse Elzaki transform of equation (17), we have

$$
\begin{aligned}
v(\zeta, \tau)= & E^{-1}\left[\left(\sum_{k=0}^{m} s^{2-\rho+k} u^{k}(\zeta, 0)+s^{\rho} E[h(\zeta, \tau)]\right)\right] \\
& -E^{-1}\left[s^{\rho} E[M v(\zeta, \tau)+N v(\zeta, \tau)]\right] .
\end{aligned}
$$

Through the iterative technique, we have

$$
v(\zeta, \tau)=\sum_{m=0}^{\infty} v_{m}(\zeta, \tau)
$$

$M$ is a linear operator:

$$
M\left(\sum_{m=0}^{\infty} v_{m}(\zeta, \tau)\right)=\sum_{m=0}^{\infty} M\left[v_{m}(\zeta, \tau)\right]
$$

and $N$ is the nonlinear function; we get

$$
\begin{aligned}
N\left(\sum_{m=0}^{\infty} v_{m}(\zeta, \tau)\right)= & v_{0}(\zeta, \tau)+M\left(\sum_{k=0}^{m} v_{k}(\zeta, \tau)\right) \\
& -N\left(\sum_{k=0}^{m} v_{k}(\zeta, \tau)\right)
\end{aligned}
$$

Substituting (19)-(21) in (18), we obtain the following solution:

$$
\begin{aligned}
\sum_{m=0}^{\infty} v_{m}(\zeta, \tau)= & E^{-1}\left[s^{\rho}\left(\sum_{k=0}^{m} s^{2-\zeta+k} u^{k}(\zeta, 0)+E[h(\zeta, \tau)]\right)\right] \\
& -E^{-1}\left[s^{\rho} E\left[M\left(\sum_{k=0}^{m} v_{k}(\zeta, \tau)\right)-N\left(\sum_{k=0}^{m} v_{k}(\zeta, \tau)\right)\right]\right] .
\end{aligned}
$$

Applying the iterative method, we get

$$
\begin{aligned}
v_{0}(\zeta, \tau) & =E^{-1}\left[s^{\rho}\left(\sum_{k=0}^{m} s^{2-\zeta+k} u^{k}(\zeta, 0)+s^{\rho} E(g(\zeta, \tau))\right)\right] \\
v_{1}(\zeta, \tau) & =-E^{-1}\left[s^{\rho} E\left[M\left[v_{0}(\zeta, \tau)\right]\right]+N\left[v_{0}(\zeta, \tau)\right]\right] \\
v_{m+1}(\zeta, \tau) & =-E^{-1}\left[s^{\rho} E\left[-M\left(\sum_{k=0}^{m} v_{k}(\zeta, \tau)\right)-N\left(\sum_{k=0}^{m} v_{k}(\zeta, \tau)\right)\right]\right], \quad m \geq 1
\end{aligned}
$$

Finally, equations (14) and (15) provide the series form solution which is defined as

$$
v(\zeta, \tau) \cong v_{0}(\zeta, \tau)+v_{1}(\zeta, \tau)+v_{2}(\zeta, \tau)+\cdots+v_{m}(\zeta, \tau)
$$$$
m \in \mathbb{N} \text {. }
$$

3.1. Error Analysis of the Projected Technique. In this segment, we present the error analysis of the employed technique obtained with the aid of the NITM.

Theorem 1. If we can find a real number $0<k<1$ satisfying $\left\|v_{m+1}(r, s)\right\| \leq k\left\|v_{m}(r, s)\right\|$ for all values of $m$ and, moreover, if 
the truncated series $\sum_{m=0}^{l} v_{m}(r, s)$ is employed as an approximate solution $v(r, s)$, then the maximum absolute truncated error can be obtained by

$$
\left\|v(r, s)-\sum_{m=0}^{l} v_{m}(r, s)\right\| \leq \frac{k^{l+1}}{(1-k)}\left\|v_{0}(r, s)\right\| .
$$

$$
\begin{aligned}
\left\|v(r, s)-\sum_{m=0}^{l} v_{m}(r, s)\right\| & =\left\|\sum_{m=l+1}^{\infty} v_{m}(r, s)\right\| \leq \sum_{m=l+1}^{\infty}\left\|v_{m}(r, s)\right\| \leq \sum_{m=l+1}^{\infty} k^{m}\left\|v_{0}(r, s)\right\| \\
& \leq(k)^{l+1}\left[1+(k)^{1}+(k)^{2}+\ldots\right]\left\|v_{0}(r, s)\right\| \leq \frac{k^{l+1}}{(1-k)}\left\|v_{0}(r, s)\right\|,
\end{aligned}
$$

which proves the theorem.

\section{Numerical Results}

Example 1. Consider the following fractional Kaup-Kupershmidt equation which is given as

$D_{\tau}^{\rho} \mu(\zeta, \tau)-15 \mu \mu_{\zeta \zeta \zeta}-15 p \mu_{\zeta} \mu_{\zeta \zeta}+45 \mu^{2} \mu_{\zeta}+\mu_{\zeta \zeta \zeta \zeta \zeta}=0$, with the initial condition

$$
\mu(\zeta, 0)=\frac{1}{4} w^{2} \lambda^{2} \sec h^{2}\left(\frac{w \zeta \lambda}{2}\right)+\frac{w^{2} \lambda^{2}}{12} .
$$

Using the Elzaki transform to (24), we obtain

$$
\begin{gathered}
\frac{1}{s^{\rho}} E[\mu(\zeta, \tau)]=\mu_{(0)}(\zeta, 0) s^{2-\rho}+E\left[-15 \mu \mu_{\zeta \zeta \zeta}-15 p \mu_{\zeta} \mu_{\zeta \zeta}+45 \mu^{2} \mu_{\zeta}+\mu_{\zeta \zeta \zeta \zeta \zeta}\right] \\
E[\mu(\zeta, \tau)]=s^{2} \mu(\zeta, 0)+s^{\rho} E\left[-15 \mu \mu_{\zeta \zeta \zeta}-15 p \mu_{\zeta} \mu_{\zeta \zeta}+45 \mu^{2} \mu_{\zeta}+\mu_{\zeta \zeta \zeta \zeta \zeta}\right] .
\end{gathered}
$$

Applying the inverse Elzaki transform of (29), we have

$$
\begin{aligned}
\mu(\zeta, \tau)= & E^{-1}\left[s^{2} \mu(\zeta, 0)\right] \\
& +E^{-1}\left[s^{\rho} E\left(-15 \mu \mu_{\zeta \zeta \zeta}-15 p \mu_{\zeta} \mu_{\zeta \zeta}+45 \mu^{2} \mu_{\zeta}+\mu_{\zeta \zeta \zeta \zeta \zeta}\right)\right] .
\end{aligned}
$$

Now, by applying the proposed semianalytical technique, we get

$$
\begin{aligned}
\mu_{0}(\zeta, \tau)= & \frac{1}{4} w^{2} \lambda^{2} \sec h^{2}\left(\frac{w \zeta \lambda}{2}\right)+\frac{w^{2} \lambda^{2}}{12}, \\
\mu_{1}(\zeta, \tau)= & E^{-1}\left[s^{\rho} E\left(-15 \mu_{(0)} \mu_{(0) \zeta \zeta \zeta}-15 p \mu_{(0) \zeta} \mu_{(0) \zeta \zeta}+45 \mu_{(0)}^{2} \mu_{(0) \zeta}+\mu_{(0) \zeta \zeta \zeta \zeta \zeta}\right)\right], \\
\mu_{1}(\zeta, \tau)= & \left(-\frac{1}{512} w^{7} \lambda^{7}\left(3843+480 p-4(209+60 p) \cosh (w \zeta \lambda)+\cosh (2 w \zeta \lambda) \operatorname{sech}^{6}\left(\frac{w \zeta \lambda}{2}\right) \tanh \left(\frac{w \zeta \lambda}{2}\right)\right)\right. \\
& \frac{\tau^{\rho}}{\Gamma(1+\rho)},
\end{aligned}
$$




$$
\begin{aligned}
\mu_{2}(\zeta, \tau)= & E^{-1}\left[s^{\rho} E\left(-15 \mu_{(1)} \mu_{(1) \zeta \zeta \zeta}-15 p \mu_{(1) \zeta} \mu_{(1) \zeta \zeta}+45 \mu_{(1)}^{2} \mu_{(1) \zeta}+\mu_{(1) \zeta \zeta \zeta \zeta \zeta}\right)\right], \\
\mu_{2}(\zeta, \tau)= & -733469760 p-3947228724+6\left(148082560 p+777305099+4358400 p^{2}\right) \cosh (w s \lambda) \\
& -20736000 p^{2}-48\left(3850520 p+18859301+124800 p^{2}\right) \cosh (2 w \zeta \lambda) \\
& +46313277 \cosh (3 w \zeta \lambda)+10287360 p \cosh (3 w \zeta \lambda)+345600 p^{2} \cosh (3 w \zeta \lambda) \\
& -305756 \cosh (4 w \zeta \lambda)-87360 p \cosh (4 w \zeta \lambda)+\cosh (5 w \zeta \lambda) \operatorname{sech}^{12}\left(\frac{w \zeta \lambda}{2}\right) \frac{w^{12} \lambda^{12} \tau^{2 \rho}}{524288 \Gamma(1+2 \rho)} \\
\vdots & \\
\mu_{n}(\zeta, \tau)= & E^{-1}\left[s^{\rho} E\left(-15 \mu_{(n)} \mu_{(n) \zeta \zeta \zeta}-15 p \mu_{(n) \zeta} \mu_{(n) \zeta \zeta}+45 \mu_{(n)}^{2} \mu_{(n) \zeta}+\mu_{(n) \zeta \zeta \zeta \zeta \zeta}\right)\right] .
\end{aligned}
$$

The series form result is

$$
\text { Therefore, we have }
$$

$$
\begin{aligned}
\mu(\zeta, \tau)= & \mu_{0}(\zeta, \tau)+\mu_{1}(\zeta, \tau)+\mu_{2}(\zeta, \tau) \\
& +\mu_{3}(\zeta, \tau)+\cdots+\mu_{n}(\zeta, \tau) .
\end{aligned}
$$

$$
\begin{aligned}
u(\zeta, \tau)= & \frac{1}{4} w^{2} \lambda^{2} \sec h^{2}\left(\frac{w \zeta \lambda}{2}\right)+\frac{w^{2} \lambda^{2}}{12}+\left(-\frac{1}{512} w^{7} \lambda^{7}(480 p+3843-4(60 p+209) \cosh (w \zeta \lambda)\right. \\
& +\cosh (2 w \zeta \lambda) \sec h^{6}\left(\frac{w \zeta \lambda}{2}\right) \tanh \left(\frac{w \zeta \lambda}{2}\right) \frac{\tau^{\rho}}{\Gamma(1+\rho)}+(-733469760 p-3947228724 \\
& -20736000 p^{2}+6\left(1480925060 p+778300098+3358400 p^{2}\right) \cosh (w s \lambda)-48 \\
& \left(3850520+18859301+124800 p^{2}\right) \cosh (2 w \zeta \lambda)+46313277 \cosh (3 w \zeta \lambda)+10287360 p \cosh (3 w \zeta \lambda) p \\
& +345600 p^{2} \cosh (3 w \zeta \lambda)-305756 \cosh (4 w \zeta \lambda)-87360 p \cosh (4 w \zeta \lambda) \\
& +\cosh (5 w \zeta \lambda) \sec h^{12}\left(\frac{w \zeta \lambda}{2}\right) \frac{w^{12} \lambda^{12} \tau^{2 \rho}}{524288 \Gamma(1+2 \rho)}+\cdots
\end{aligned}
$$

For $\rho=1$, the exact results of (27) are given by

$$
\mu(\zeta, \tau)=\frac{1}{4} w^{2} \lambda^{2} \sec h^{2}\left(\frac{\lambda}{2}\left(\frac{-w^{5}\left(-8 \lambda^{2} \nu+16 \nu^{2}+\lambda^{4}\right)}{16 \Gamma(1+\rho)} \tau^{\rho}+w \zeta\right)\right)+\frac{w^{2} \lambda^{2}}{12} .
$$

Analytical approximate solutions with some free parameters are provided by the proposed technique. The analytical findings are extremely useful in deciphering the internal components of acts of nature. Depending on the physical factors, the explicit solutions represented several forms of approximate solutions. Figure 1 compares the result obtained by the help of the proposed technique to the exact and analytical result for the fractional-order KK equation.
Figure 2 shows different fractional orders of $\rho$ with respect to $\zeta$ and $\tau$ comparison show that they have close contact with each other. Figure 3 shows the error plot of three- and twodimensional graphs.

Example 2. Consider the following fractional Kaup-Kupershmidt equation which is given as 

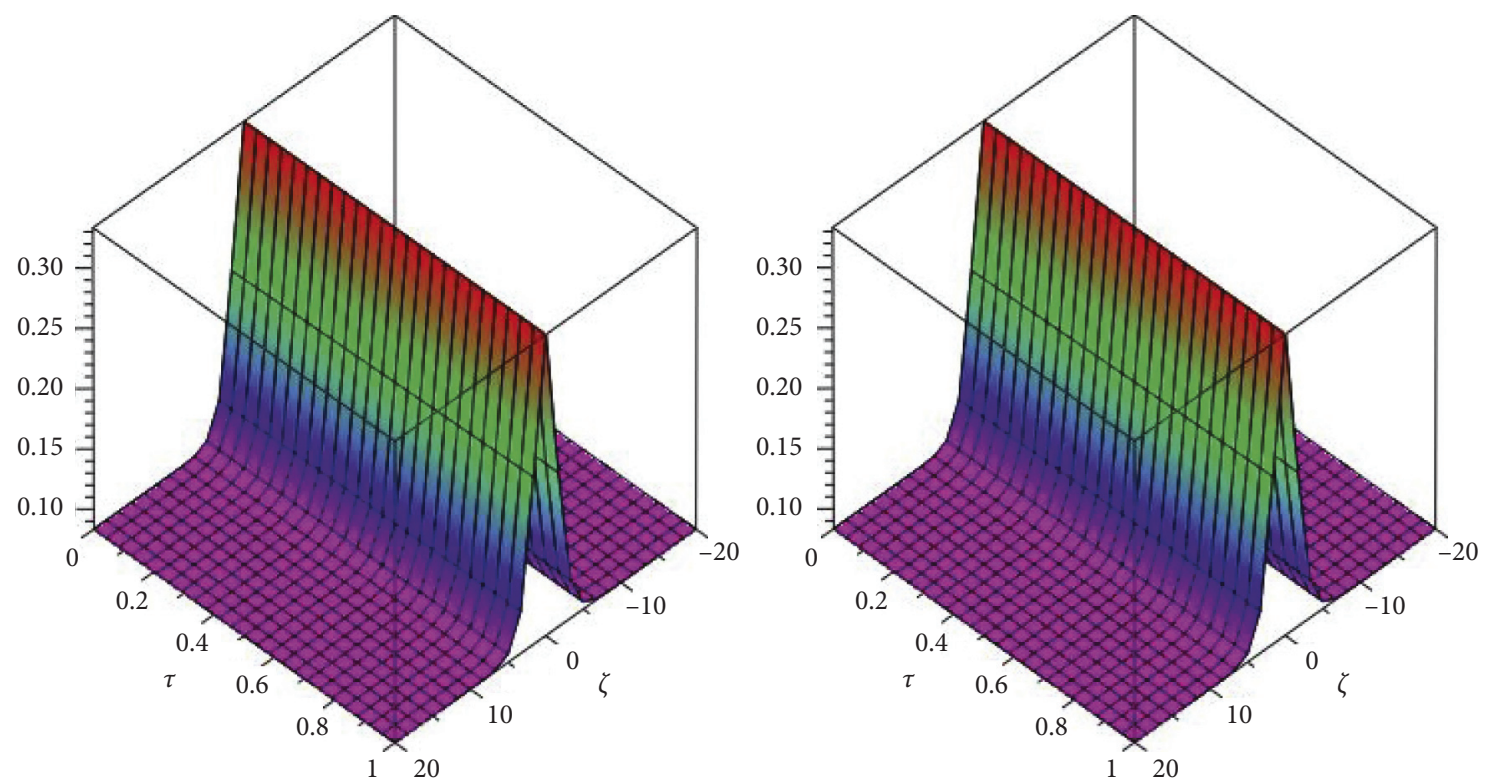

Figure 1: The exact and analytical solutions of Example 1.
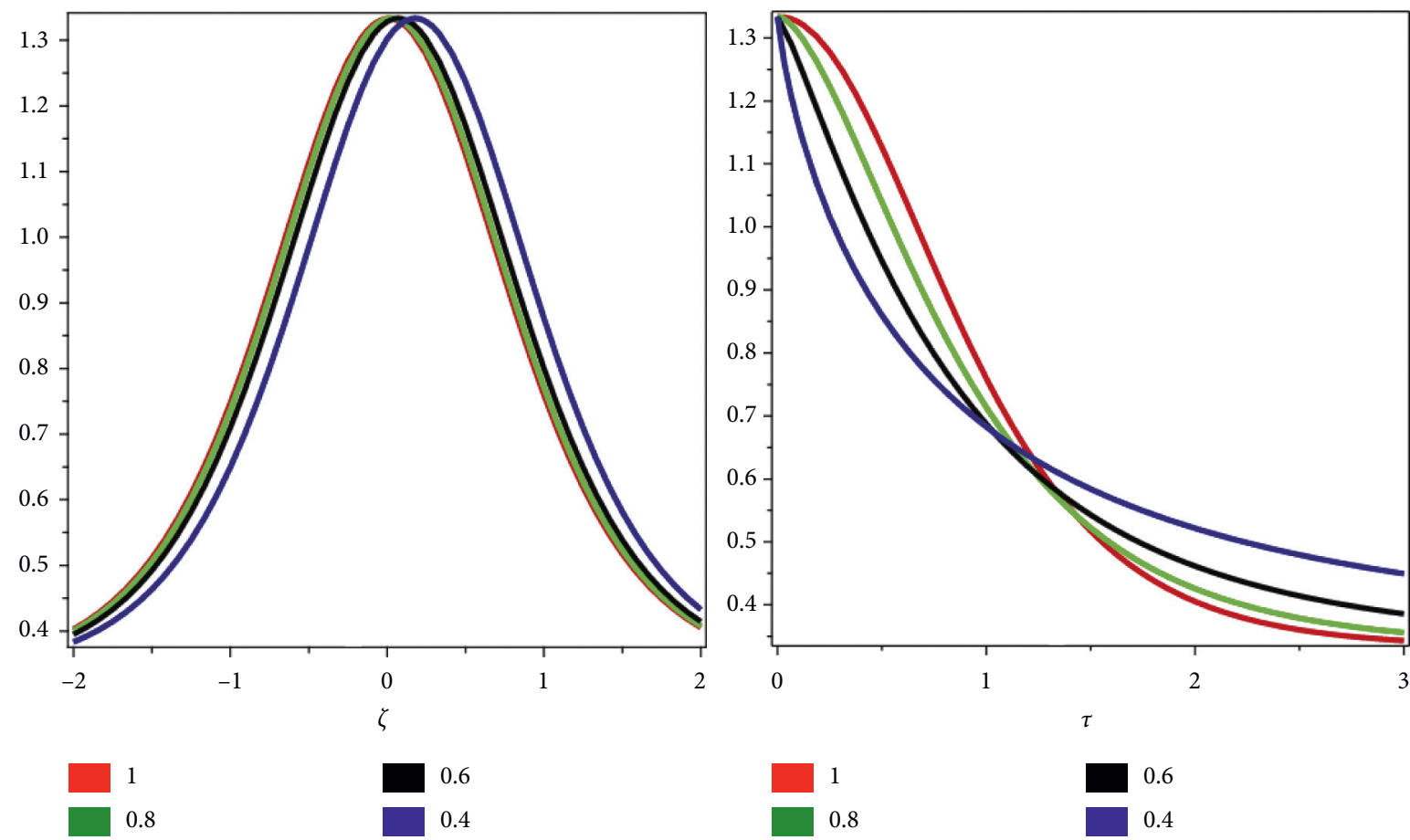

Figure 2: The fractional order $\rho$ of Example 1 with respect to $\zeta$ and $\tau$.

$$
D_{\tau}^{\rho} \mu(\zeta, \tau)-15 \mu \mu_{\zeta \zeta \zeta}-15 p \mu_{\zeta} \mu_{\zeta \zeta}+45 \mu^{2} \mu_{\zeta}+\mu_{\zeta \zeta \zeta \zeta \zeta}=0
$$

with the initial condition

$$
\mu(\zeta, 0)=\frac{4}{3} c-\frac{4}{p} \csc h^{2}(\sqrt{c \zeta}) .
$$

Using the Elzaki transform to (35), we get 

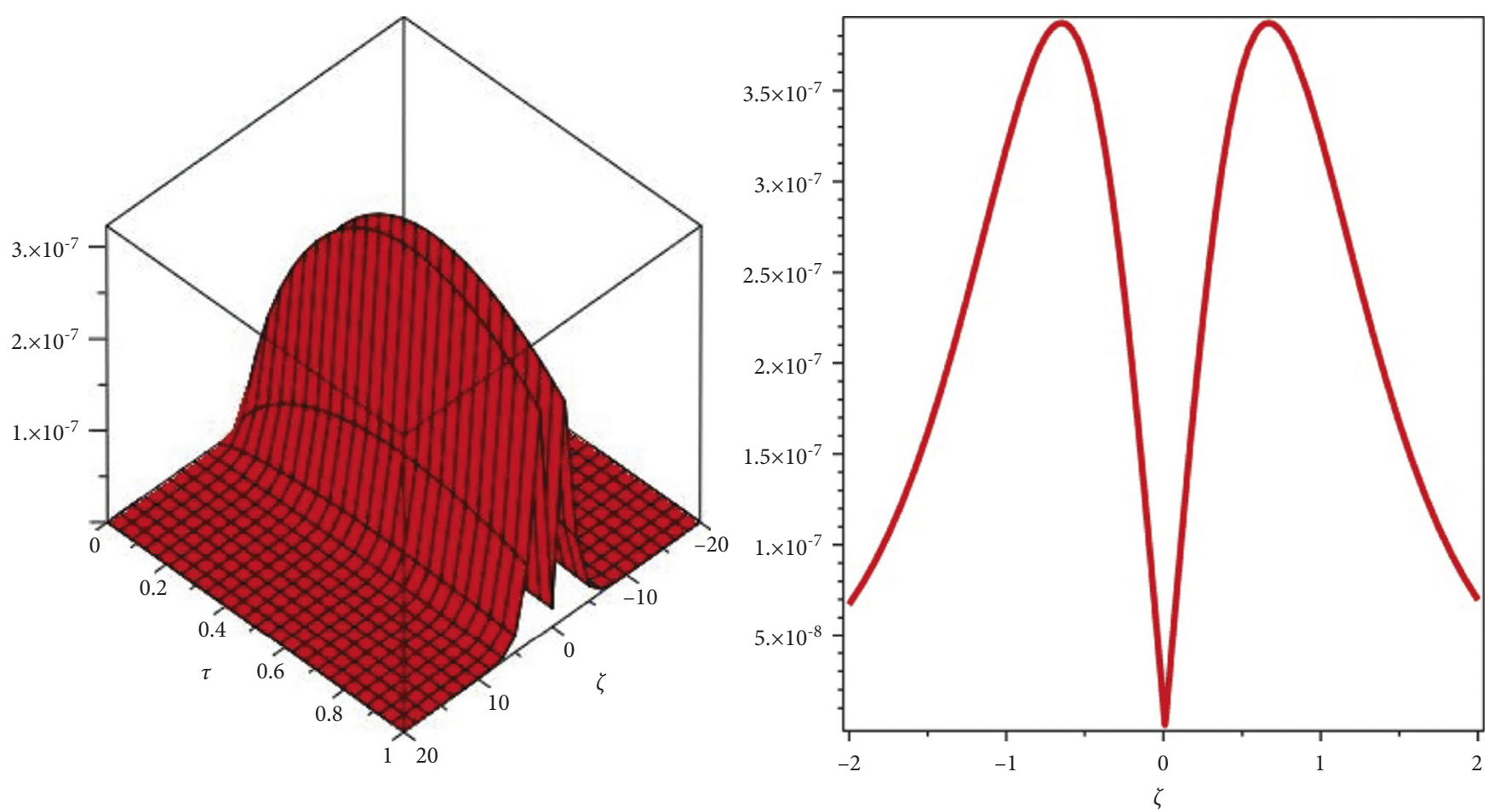

Figure 3: The 2D and 3D error plot of Problem 1.

$$
\begin{gathered}
\frac{1}{s^{\rho}} E[\mu(\zeta, \tau)]=\mu_{(0)}(\zeta, 0) s^{2-\rho}+E\left[-15 \mu \mu_{\zeta \zeta \zeta}-15 p \mu_{\zeta} \mu_{\zeta \zeta}+45 \mu^{2} \mu_{\zeta}+\mu_{\zeta \zeta \zeta \zeta \zeta}\right] \\
E[\mu(\zeta, \tau)]=s^{2} \mu(\zeta, 0)+s^{\rho} E\left[-15 \mu \mu_{\zeta \zeta \zeta}-15 p \mu_{\zeta} \mu_{\zeta \zeta}+45 \mu^{2} \mu_{\zeta}+\mu_{\zeta \zeta \zeta \zeta \zeta}\right] .
\end{gathered}
$$

Applying the inverse Elzaki transform of (38), we have

$$
\mu(\zeta, \tau)=E^{-1}\left[s^{2} \mu(\zeta, 0)\right]+E^{-1}\left[s^{\rho} E\left(-15 \mu \mu_{\zeta \zeta \zeta}-15 p \mu_{\zeta} \mu_{\zeta \zeta}+45 \mu^{2} \mu_{\zeta}+\mu_{\zeta \zeta \zeta \zeta \zeta}\right)\right] .
$$

Now, by applying the proposed semianalytical technique, we get

$$
\begin{aligned}
\mu_{0}(\zeta, \tau)= & \frac{4}{3} c-\frac{4}{p} \operatorname{csch}^{2}(\sqrt{c \zeta}), \\
\mu_{1}(\zeta, \tau)= & E^{-1}\left[s^{\rho} E\left(-15 \mu_{(0)} \mu_{(0) \zeta \zeta \zeta}-15 p \mu_{(0) \zeta} \mu_{(0) \zeta \zeta}+45 \mu_{(0)}^{2} \mu_{(0) \zeta}+\mu_{(0) \zeta \zeta \zeta \zeta \zeta}\right)\right] . \\
\mu_{1}(\zeta, \tau)= & 63 p^{2}+360-420 p+4 p(16 p-15) \cosh (2 \sqrt{c \zeta}) \\
& +p^{2} \cosh (4 \sqrt{c x}) \operatorname{sech}^{6}(\sqrt{c \zeta}) \tanh (\sqrt{c \zeta}) \frac{16 c^{7 / 2} \tau^{\rho}}{p^{3} \Gamma(1+\rho)}, \\
\mu_{2}(\zeta, \tau)= & E^{-1}\left[s^{\rho} E\left(-15 \mu_{(1)} \mu_{(1) \zeta \zeta \zeta}-15 p \mu_{(1) \zeta} \mu_{(1) \zeta \zeta}+45 \mu_{(1)}^{2} \mu_{(1) \zeta}+\mu_{(1) \zeta \zeta \zeta \zeta \zeta}\right)\right] .
\end{aligned}
$$




$$
\begin{aligned}
\mu_{2}(\zeta, \tau)= & -306084 p^{4}-3110400+14515200 p-26369280 p^{3} \\
& -6\left(2217600 p-432000+2656400 p^{3}-4451160 p^{2}+9181 p^{4}\right) \\
& \cosh (2 \sqrt{c \zeta})+48 p\left(41590 p^{2}+14400-60780 p+4789 p^{3}\right) \\
& \cosh (4 \sqrt{c \zeta})-59040 p^{3} \cosh (6 \sqrt{c \zeta})+79920 p^{2} \cosh (6 \sqrt{c \zeta})-20883 p^{4} \cosh (6 \sqrt{c \zeta}) \\
& -240 p^{3} \cosh (8 \sqrt{c \zeta})+p^{4} \cosh (10 \sqrt{c \zeta})+244 p^{4} \cosh (8 \sqrt{c \zeta}) \frac{8 c^{2} \tau^{2 \rho} \operatorname{sech}^{12} \sqrt{c \zeta}}{p^{5} \Gamma(1+2 \rho)} \\
\vdots & \\
\mu_{n}(\zeta, \tau)= & E^{-1}\left[s^{\rho} E\left(-15 \mu_{(n)} \mu_{(n) \zeta \zeta \zeta}-15 p \mu_{(n) \zeta} \mu_{(n) \zeta \zeta}+45 \mu_{(n)}^{2} \mu_{(n) \zeta}+\mu_{(n) \zeta \zeta \zeta \zeta \zeta}\right)\right] .
\end{aligned}
$$

The series form result is

Therefore, we have

$$
\begin{aligned}
\mu(\zeta, \tau)= & \mu_{0}(\zeta, \tau)+\mu_{1}(\zeta, \tau)+\mu_{2}(\zeta, \tau)+\mu_{3}(\zeta, \tau) \\
& +\cdots+\mu_{n}(\zeta, \tau) .
\end{aligned}
$$

$$
\begin{aligned}
u(\zeta, \tau)= & \frac{4}{3} c-\frac{4}{p} \csc h^{2}(\sqrt{c \zeta})+\left(63 p^{2}+360-420 p+4 p(16 p-15) \cosh (2 \sqrt{c \zeta})\right. \\
& +p^{2} \cosh (4 \sqrt{c x}) \sec h^{6}(\sqrt{c \zeta}) \tanh (\sqrt{c \zeta}) \frac{16 c^{7 / 2} \tau^{\rho}}{p^{3} \Gamma(1+\rho)} \\
& +\left\{14515200 p-3110400-306084 p^{4}-26369280 p^{3}\right. \\
& -6\left(2656400 p^{3}+2217600 p-4451160 p^{2}-432000+9181 p^{4}\right) \cosh (2 \sqrt{c \zeta}) \\
& +48 p\left(41590 p^{2}+14400+4789 p^{3}-60780 p\right) \cosh (4 \sqrt{c \zeta})+79920 p^{2} \cosh (6 \sqrt{c \zeta}) \\
& -59040 p^{3} \cosh (6 \sqrt{c \zeta})-20883 p^{4} \cosh (6 \sqrt{c \zeta})-240 p^{3} \cosh (8 \sqrt{c \zeta}) \\
& \left.+p^{4} \cosh (10 \sqrt{c \zeta})+244 p^{4} \cosh (8 \sqrt{c \zeta})\right\} \frac{8 c^{2} \tau^{2 \rho} \sec h^{12} \sqrt{c \zeta}}{p^{5} \Gamma(1+2 \rho)}+\cdots
\end{aligned}
$$

For $\rho=1$, the exact results of (35) are given by

$$
\mu(\zeta, \tau)=\frac{4}{3} c-\frac{4}{p} \operatorname{sech}^{2}\left(\sqrt{c}\left(\zeta+8\left(3 c^{2}-5 p c\right) \tau\right)\right)
$$

Analytical approximate solutions with some free parameters are provided by the proposed technique. The analytical findings are extremely useful in deciphering the internal components of acts of nature. Depending on the physical factors, the explicit solutions represented several forms of approximate solutions. Figure 4 compares the result obtained by the help of the proposed technique to the exact and analytical result for the fractional-order KK equation. Figure 5 shows different fractional orders of $\rho$ with respect to $\zeta$ and $\tau$ comparison which show that they have close contact with each other.

Example 3. Consider the following fractional Kaup-Kupershmidt equation which is given as

$$
D_{\tau}^{\alpha} \mu(\zeta, \tau)=5 \mu \mu_{\zeta \zeta \zeta}+\frac{25}{2} \mu_{\zeta} \mu_{\zeta \zeta}+5 \mu^{2} \mu_{\zeta}+\mu_{\zeta \zeta \zeta \zeta \zeta}
$$

with the initial condition

$$
\mu(\zeta, 0)=-2 k^{2}+\frac{24 k^{2}}{1+e^{k \zeta}} c-\frac{24 k^{2}}{1+e k \zeta} .
$$

Using the Elzaki transform to (44), we get 

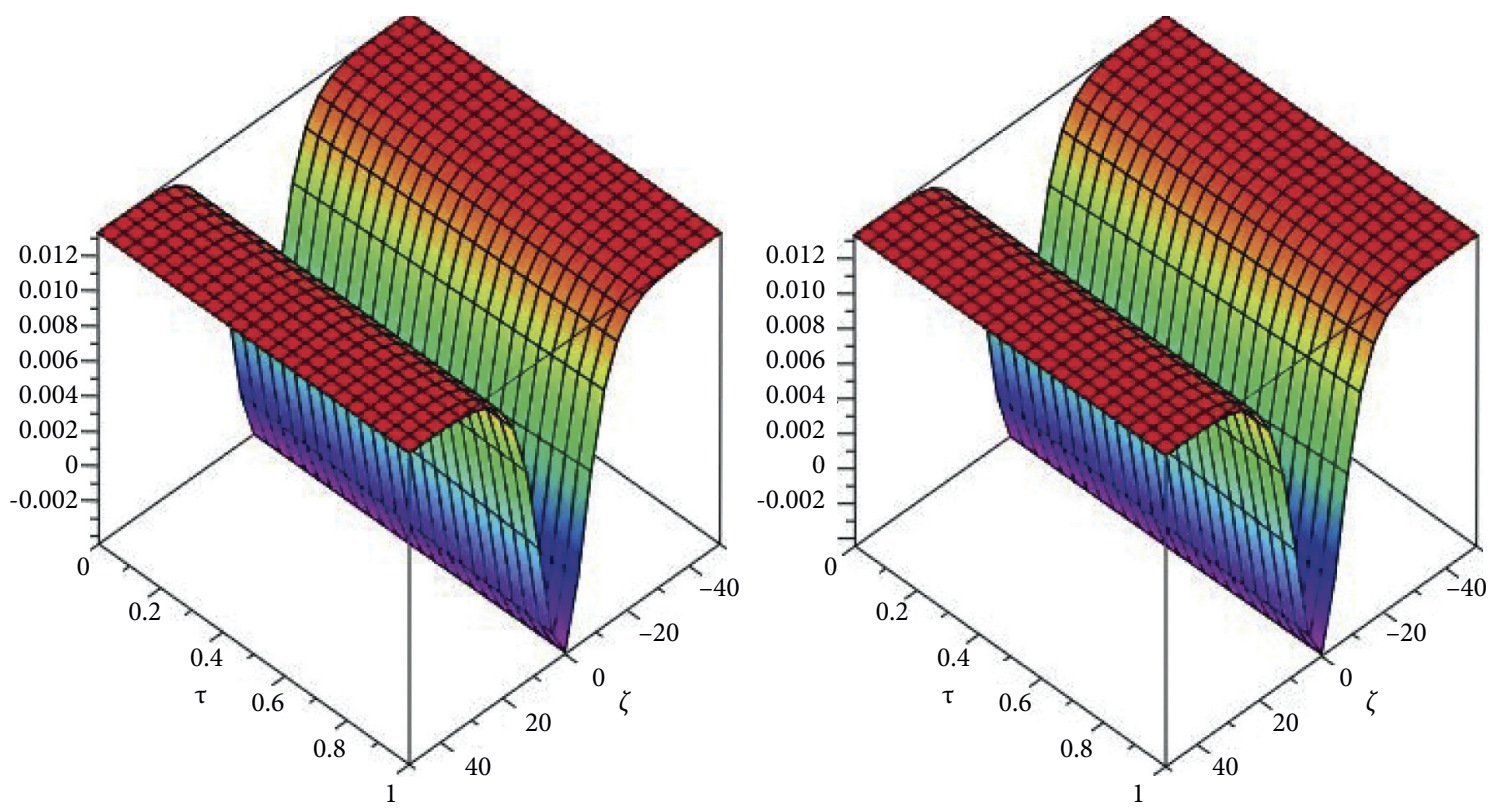

FIgURE 4: The exact and analytical solutions of Example 2.
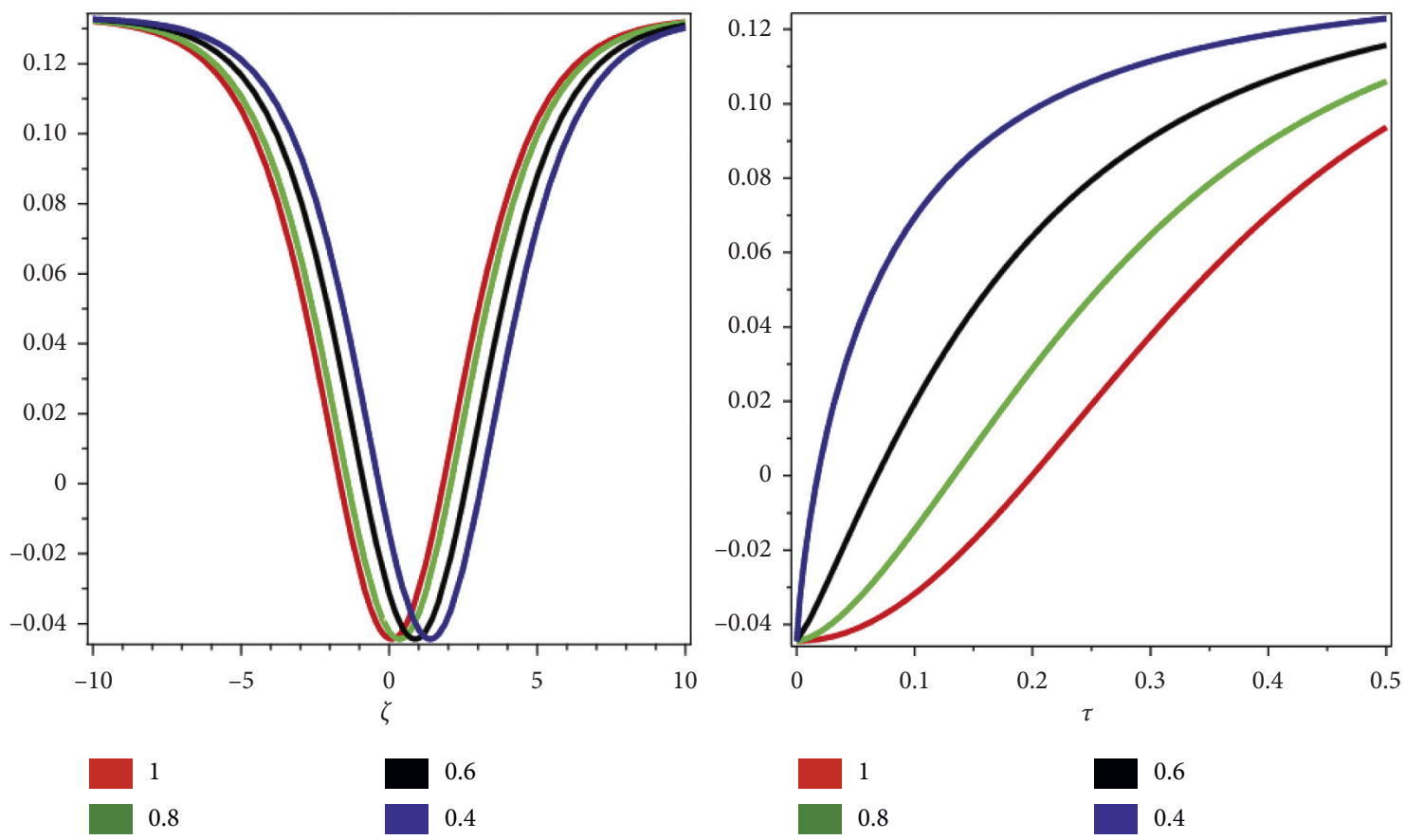

Figure 5: The fractional order $\rho$ of Example 2 with respect to $\zeta$ and $\tau$.

$$
\begin{gathered}
\frac{1}{s^{\rho}} E[\mu(\zeta, \tau)]=\mu_{(0)}(\zeta, 0) s^{2-\rho}+E\left[5 \mu \mu_{\zeta \zeta \zeta}+\frac{25}{2} \mu_{\zeta} \mu_{\zeta \zeta}+5 \mu^{2} \mu_{\zeta}+\mu_{\zeta \zeta \zeta \zeta \zeta}\right] \\
E[\mu(\zeta, \tau)]=s^{2} \mu(\zeta, 0)+s^{\rho} E\left[5 \mu \mu_{\zeta \zeta \zeta}+\frac{25}{2} \mu_{\zeta} \mu_{\zeta \zeta}+5 \mu^{2} \mu_{\zeta}+\mu_{\zeta \zeta \zeta \zeta \zeta}\right] .
\end{gathered}
$$


10

Journal of Mathematics
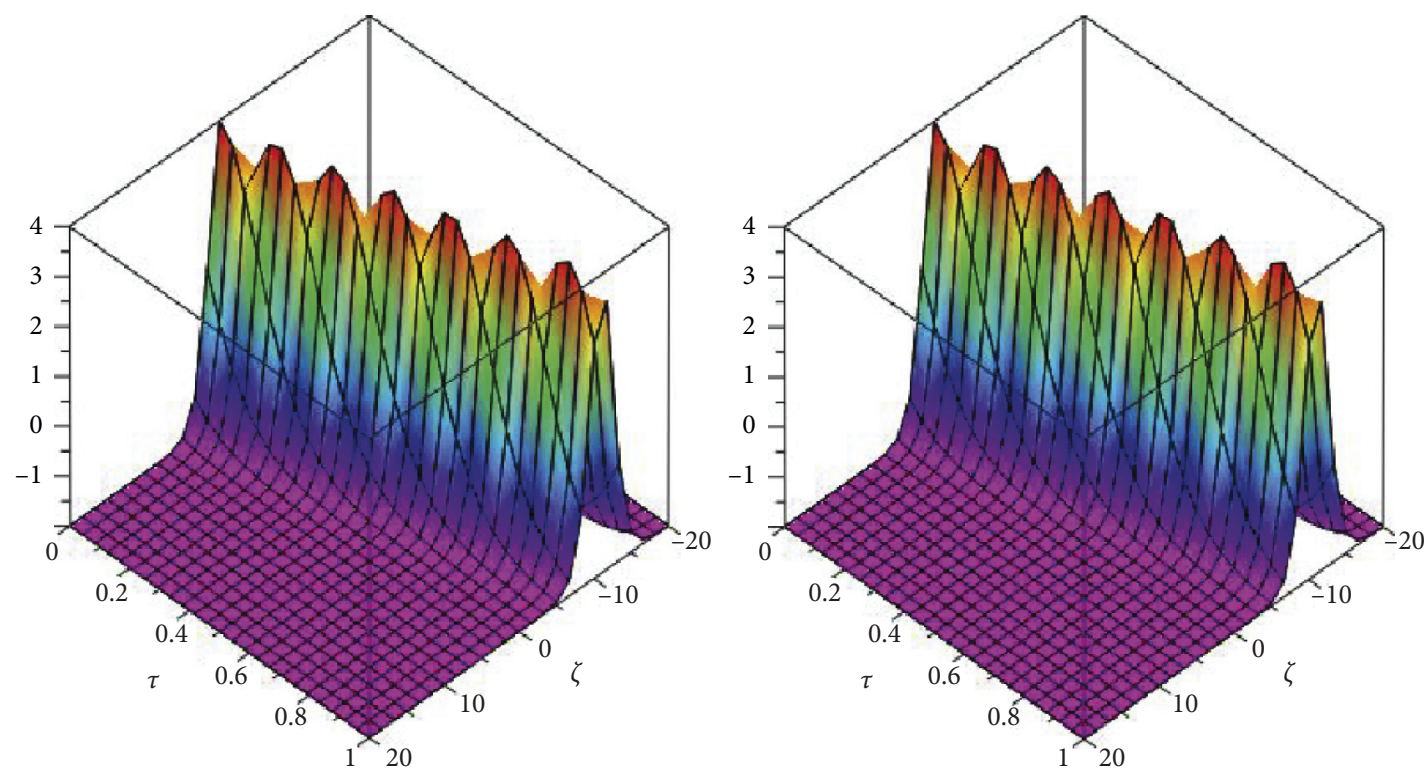

Figure 6: The exact and analytical solutions of Example 3.

Applying the inverse Elzaki transformation of (47), we have

$$
m u(\zeta, \tau)=E^{-1}\left[s^{2} \mu(\zeta, 0)\right]+E^{-1}\left[s^{\rho} E\left(5 \mu \mu_{\zeta \zeta \zeta}+\frac{25}{2} \mu_{\zeta} \mu_{\zeta \zeta}+5 \mu^{2} \mu_{\zeta}+\mu_{\zeta \zeta \zeta \zeta \zeta}\right)\right] .
$$

Now, by applying the proposed semianalytical technique, we get

$$
\begin{aligned}
& \mu_{0}(\zeta, \tau)=-2 k^{2}+\frac{24 k^{2}}{1+e^{k \zeta}} c-\frac{24 k^{2}}{1+e k \zeta}, \\
& \mu_{1}(\zeta, \tau)=E^{-1}\left[s^{\rho} E\left(5 \mu_{(0)} \mu_{(0) \zeta \zeta \zeta}+\frac{25}{2} \mu_{(0) \zeta} \mu_{(0) \zeta \zeta}+5 \mu_{(0)}^{2} \mu_{(0) \zeta}+\mu_{(0) \zeta \zeta \zeta \zeta \zeta}\right)\right] . \\
& \mu_{1}(\zeta, \tau)=\frac{\tau^{\rho}}{\Gamma(1+\rho)}\left(\frac{264 e^{k \zeta}\left(-1+e^{k \zeta}\right) k^{7}}{\left(1+e^{k \zeta}\right)^{3}}\right) \\
& \mu_{2}(\zeta, \tau)=E^{-1}\left[s^{\rho} E\left(5 \mu_{(1)} \mu_{(1) \zeta \zeta \zeta}+\frac{25}{2} \mu_{(1) \zeta} \mu_{(1) \zeta \zeta}+5 \mu_{(1)}^{2} \mu_{(1) \zeta}+\mu_{(1) \zeta \zeta \zeta \zeta \zeta}\right)\right] . \\
& \mu_{2}(\zeta, \tau)=\frac{2904 e^{k \zeta}\left(1-4 e^{k \zeta}+e^{2 k \zeta}\right) k^{12} \tau^{2 \rho}}{\left(1+e^{k \zeta}\right)^{4} \Gamma(1+2 \rho)} \\
& \mu_{3}(\zeta, \tau)=E^{-1}\left[s^{\rho} E\left(5 \mu_{(2)} \mu_{(2) \zeta \zeta \zeta}+\frac{25}{2} \mu_{(2) \zeta} \mu_{(2) \zeta \zeta}+5 \mu_{(2)}^{2} \mu_{(2) \zeta}+\mu_{(2) \zeta \zeta \zeta \zeta \zeta}\right)\right] .
\end{aligned}
$$




$$
\begin{aligned}
\mu_{3}(\zeta, \tau)= & 2904 e^{k \zeta}\left(-1+e^{k \zeta}\right) k^{17} \tau^{3 \rho}\left(\left(11+54 e^{k \zeta}-4923 e^{2 k \zeta}+10228 e^{3 k \zeta}-4923 e^{4 k \zeta}+54 e^{5 k \zeta}+11 e^{6 k \zeta}\right)\right. \\
& \Gamma(1+\rho)^{2}-60 e^{k \zeta}\left(1-38 e^{k \zeta}+90 e^{2 k \zeta}-38 e^{3 k \zeta}+e^{4 k \zeta}\right) \\
& \Gamma(1+2 \rho) \div\left(1+e^{k \zeta}\right)^{9} \Gamma(1+\rho)^{2} \Gamma(1+3 \rho) \\
\vdots & \\
\mu_{n}(\zeta, \tau)= & E^{-1}\left[s^{\rho} E\left(5 \mu_{(n)} \mu_{(n) \zeta \zeta \zeta}+\frac{25}{2} \mu_{(n) \zeta} \mu_{(n) \zeta \zeta}+5 \mu_{(n)}^{2} \mu_{(n) \zeta}+\mu_{(n) \zeta \zeta \zeta \zeta \zeta}\right)\right] .
\end{aligned}
$$

The series form result is

$$
\mu(\zeta, \tau)=\mu_{0}(\zeta, \tau)+\mu_{1}(\zeta, \tau)+\mu_{2}(\zeta, \tau)+\mu_{3}(\zeta, \tau)+\cdots+\mu_{n}(\zeta, \tau)
$$

Therefore, we have

$$
\begin{aligned}
u(\zeta, \tau)= & -2 k^{2}+\frac{24 k^{2}}{1+e^{k \zeta}} c-\frac{24 k^{2}}{1+e k \zeta}+\frac{\tau^{\rho}}{\Gamma(1+\rho)}\left(\frac{264 e^{k \zeta}\left(-1+e^{k \zeta}\right) k^{7}}{\left(1+e^{k \zeta}\right)^{3}}\right) \\
& +\frac{2904 e^{k \zeta}\left(1-4 e^{k \zeta}+e^{2 k \zeta}\right) k^{12} \tau^{2 \rho}}{\left(1+e^{k \zeta}\right)^{4} \Gamma(1+2 \rho)}+2904 e^{k \zeta}\left(-1+e^{k \zeta}\right) k^{17} \tau^{3 \rho} \\
& \left(11+54 e^{k \zeta}-4923 e^{2 k \zeta}+10228 e^{3 k \zeta}-4923 e^{4 k \zeta}+54 e^{5 k \zeta}+11 e^{6 k \zeta}\right) \\
& \Gamma(1+\rho)^{2}-60 e^{k \zeta}\left(1-38 e^{k \zeta}+90 e^{2 k \zeta}-38 e^{3 k \zeta}+e^{4 k \zeta}\right) \\
& \Gamma(1+2 \rho) \div\left(1+e^{k \zeta}\right)^{9} \Gamma(1+\rho)^{2} \Gamma(1+3 \rho)+\cdots
\end{aligned}
$$

For $\rho=1$, the exact results of (44) are given by

$$
\mu(\zeta, \tau)=-2 k^{2}+\frac{24 k^{2}}{1+e^{k \zeta+11 k^{5} \tau}}-\frac{24 k^{2}}{\left(1+e^{k \zeta+11 k^{5} \tau}\right)^{2}} .
$$

Analytical approximate solutions with some free parameters are provided by the proposed technique. The analytical findings are extremely useful in deciphering the internal components of acts of nature. Depending on the physical factors, the explicit solutions represented several forms of approximate solutions. Figure 6 compares the result obtained by the help of the proposed technique to the exact and analytical result for the fractional-order KK equation. Figure 7 shows different fractional orders of $\rho$ with respect to $\zeta$ and $\tau$ comparison which show that they have close contact with each other. 

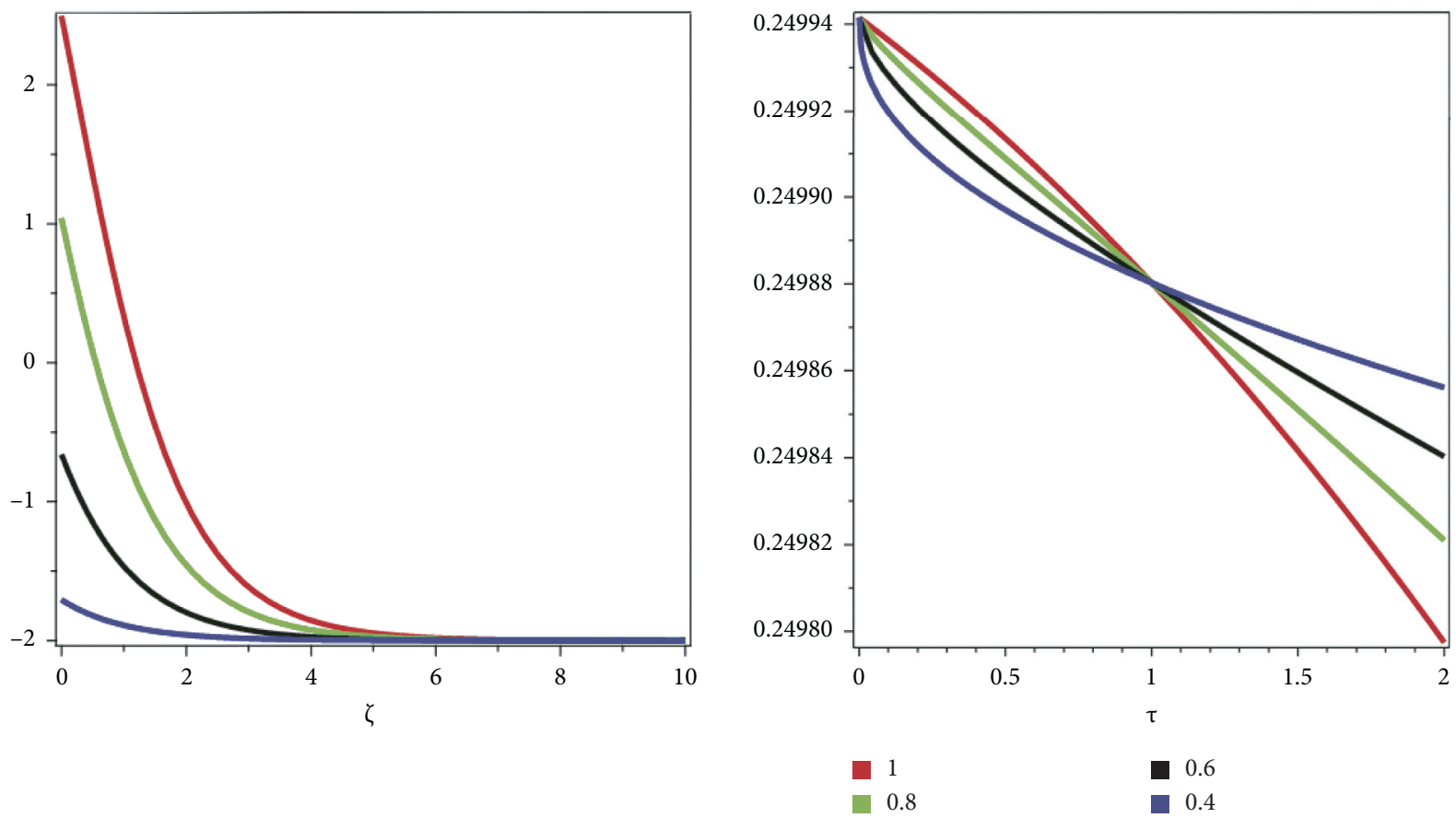

Figure 7: The fractional order $\rho$ of Example 3 with respect to $\zeta$ and $\tau$.

\section{Conclusion}

In this article, the iterative transformation technique is utilized to achieve analytical solutions of the fractional-order Kaup-Kupershmidt equations, which are broadly utilized as problems for spatial effects in applied sciences. The method gave a series type of solutions that converge very quickly in the mathematical model. It is predicted that the results obtained in this paper will be effective for more evaluation of the complicated nonlinear physical models. The analyses of this method are very clear and straightforward. As a result, we conclude that this method can be used to solve a variety of nonlinear fractional-order partial differential equation schemes.

\section{Data Availability}

The numerical data used to support the findings of this study are included within the article.

\section{Conflicts of Interest}

The authors declare that there are no conflicts of interest regarding the publication of this article.

\section{Acknowledgments}

This research was funded by Scientific Research Deanship at the University of Ha'il, Saudi Arabia, through project no. RG-21 005.

\section{References}

[1] J. H. He, "Nonlinear oscillation with fractional derivative and its applications," International conference on vibrating engineering, vol. 98, pp. 288-291, 1998.
[2] R. Almeida, N. R. O. Bastos, and M. T. T. Monteiro, "Modeling some real phenomena by fractional differential equations," Mathematical Methods in the Applied Sciences, vol. 39, no. 16, pp. 4846-4855, 2016.

[3] Y. A. Rossikhin and M. V. Shitikova, "Application of fractional calculus for dynamic problems of solid mechanics: novel trends and recent results," Applied Mechanics Reviews, vol. 63, no. 1, 2010.

[4] Y. A. Rossikhin, "Reflections on two parallel ways in the progress of fractional calculus in mechanics of solids," Applied Mechanics Reviews, vol. 63, no. 1, 2010.

[5] P. Sunthrayuth, A. M. Zidan, S.-W. Yao, R. Shah, and M. Inc, "The comparative study for solving fractional-order fornbergwhitham equation via $\rho$-laplace transform," Symmetry, vol. 13, no. 5 , p. $784,2021$.

[6] R. P. Agarwal, F. Mofarreh, R. Shah, W. Luangboon, and K. Nonlaopon, "An analytical technique, based on natural transform to solve fractional-order parabolic equations," Entropy, vol. 23, no. 8, p. 1086, 2021.

[7] Y. Zhang, H. Sun, H. H. Stowell, M. Zayernouri, and S. E. Hansen, "A review of applications of fractional calculus in Earth system dynamics," Chaos, Solitons \& Fractals, vol. 102, pp. 29-46, 2017.

[8] N. H. Aljahdaly, R. P. Agarwal, R. Shah, and T. Botmart, "Analysis of the time fractional-order coupled burgers equations with non-singular kernel operators," Mathematics, vol. 9, no. 18 , p. 2326, 2021.

[9] F. Mohammadi, L. Moradi, D. Baleanu, and A. Jajarmi, "A hybrid functions numerical scheme for fractional optimal control problems: application to nonanalytic dynamic systems," Journal of Vibration and Control, vol. 24, no. 21, pp. 5030-5043, 2018.

[10] A. Jajarmi and D. Baleanu, "On the fractional optimal control problems with a general derivative operator," Asian Journal of Control, vol. 23, 2019.

[11] D. J. Kaup, "On the inverse scattering for cubic eigenvalue problems of the class equations," Studies in Applied Mathematics, vol. 62, pp. 183-195, 1980. 
[12] B. A. Kupershmidt, "A super Korteweg-de-Vries equations: an integrable system," Physics Letters A, vol. 102, pp. 213-218, 1994.

[13] E. Fan, "Uniformly constructing a series of explicit exact solutions to nonlinear equations in mathematical physics," Chaos, Solitons \& Fractals, vol. 16, no. 5, pp. 819-839, 2003.

[14] M. Inc, "On numerical soliton solution of the Kaup-Kupershmidt equation and convergence analysis of the decomposition method," Applied Mathematics and Computation, vol. 172, no. 1, pp. 72-85, 2006.

[15] K. Nonlaopon, A. M. Alsharif, A. M. Zidan, A. Khan, Y. S. Hamed, and R. Shah, "Numerical investigation of fractional-order Swift-Hohenberg equations via a Novel transform," Symmetry, vol. 13, no. 7, p. 1263, 2021.

[16] H.-W. Tam and X.-B. Hu, "Two integrable differential-difference equations exhibiting soliton solutions of the KaupKupershmidt equation type," Physics Letters A, vol. 272, no. 3 , pp. 174-183, 2000.

[17] M. Musette and C. Verhoeven, "Nonlinear superposition formula for the Kaup-Kupershmidt partial differential equation," Physica D: Nonlinear Phenomena, vol. 144, no. 1-2, pp. 211-220, 2000.

[18] D. G. Prakasha, N. S. Malagi, P. Veeresha, and B. C. Prasannakumara, "An efficient computational technique for time-fractional Kaup-Kupershmidt equation," Numerical Methods for Partial Differential Equations, vol. 37, pp. 1-18, 2020.

[19] V. Daftardar-Gejji and H. Jafari, "An iterative method for solving nonlinear functional equations," Journal of Mathematical Analysis and Applications, vol. 316, no. 2, pp. 753-763, 2006.

[20] H. Jafari, Iterative Methods for Solving System of Fractional Differential Equations, Ph.D. Thesis, Pune University, Pune, Maharashtra, 2006.

[21] H. Jafari, M. Nazari, D. Baleanu, and C. M. Khalique, "A new approach for solving a system of fractional partial differential equations," Computers \& Mathematics with Applications, vol. 66, no. 5, pp. 838-843, 2013.

[22] A. Prakash, M. Kumar, and D. Baleanu, "A new iterative technique for a fractional model of nonlinear ZakharovKuznetsov equations via Sumudu transform," Applied Mathematics and Computation, vol. 334, pp. 30-40, 2018.

[23] K. Nonlaopon, M. Naeem, A. M. Zidan, R. Shah, A. Alsanad, and A. Gumaei, "Numerical investigation of the time-fractional whitham-broer-kaup equation involving without singular kernel operators," Complexity, vol. 2021, Article ID 7979365, 21 pages, 2021.

[24] M. Ramadan and M. S. Al-luhaibi, "New iterative method for solving the Fornberg-Whitham equation and comparison with homotopy perturbation transform method," British Journal of Mathematics \& Computer Science, vol. 4, no. 9, pp. 1213-1227, 2014.

[25] T. M. Elzaki, "The new integral transform Elzaki transform," Global Journal of Pure and Applied Mathematics, vol. 7, no. 1, pp. 57-64, 2011.

[26] T. M. Elzaki, "On the connections between Laplace and Elzaki transforms," Advances in Theoretical and Applied Mathematics, vol. 6, no. 1, pp. 1-11, 2011.

[27] T. M. Elzaki, "On the new integral transform Elzaki Transform" fundamental properties investigations and applications," Global Journal of Mathematical Sciences: Theory and Practical, vol. 4, no. 1, pp. 1-13, 2012. 